\title{
The Order of Edwards and Montgomery Curves
}

\author{
RUSLAN SKURATOVSKII \\ Department of Computer Science \\ University Igor Sikorsky Kiev Polytechnic Institute, National Technical University of Ukraine \\ Peremogy 37 \\ UKRAINE
}

VOLODYMYR OSADCHYY

ceo IT-GRAVITY-VO, Inc.

Orlando, Florida, Edgewater Sr, Suite 1888, Orlando, FL, 32804,

USA

Abstract: - The Elliptic Curve Digital Signature Algorithm (ECDSA) is the elliptic curve analogue of the Digital Signature Algorithm (DSA) [2]. It is well kn own that the problem of discrete logarithm is NP-hard on group on elliptic curve (EC) [5]. The orders of groups of an algebraic affine and projective curves of Edwards $[3,9]$ over the finite field $\mathrm{F}_{p^{n}}$ is studied by us. We research Edwards algebraic curves over a finite field, which are one of th e most promising supports of sets of points which are used for fast group operations [ 1]. We construct a new method for counting the order of a $\mathrm{n}$ Edwards curve $E_{d}\left[\mathrm{~F}_{p}\right]$ over a finite field $\mathrm{F}_{p}$. It should be noted that this method can be applied to the order of elliptic curves due to the birational equivalence between elliptic curves and Edwar ds curves. The method we have proposed has much less complexity $O\left(p \log _{2}^{2} p\right)$ at not large values $p$ in comparison with the best Schoof basic algorithm with complexity $O\left(\log _{2}^{8} p^{n}\right)$, as well as a variant of the Schoof algorithm that uses fast arithmetic, which has complexity $O\left(\log _{2}^{4} p^{n}\right)$, but works only for Elkis or Atkin primes. We not only find a specific set of coefficients with corresponding field characteristics for which these curves are su persingular, but we additionally find a general for mula by which one can determine whether a curve $E_{d}\left[\mathrm{~F}_{p}\right]$ is supersingular over this field or not. The symmetric of the Edwards curve form and the parity of all degrees made it possible to represent the shape curves and apply the method of calculating the residual coincidences.

A birational isomorphism between the Montgomery curve and the Edwards curve is also constructed. A oneto-one correspondence between the Ed wards supersingular curves and Montg omery supersingular curves is established. The criterion of supersingularity for Edwards curves is found over $\mathrm{F}_{p^{n}}$.

Key-Words: - finite field, elliptic curve, Edwards curve, algor ithm of order counting of group of points of an elliptic curve.

Received: October 19, 2019. Revised: May 7, 2020. Re-revised: May 22, 2020. Accepted: May 26, 2020.

Published: May 28, 2020.

\section{Introduction}

The method of finding th $\mathrm{e}$ order of an algebraic curve over a finite fiel $\mathrm{d}_{p^{n}}$ are relat ed with constructing of curves of given order. To construct cryptosystem based on ellip tic curve we need to analyze the order of a group of el liptic curve points. Our method gives an approach to co nstruct Edwards curves of determined order that if very important if cryptography and coding theory. It was accepted in 1999 as an ANSI standard and in 2000 as IEEE and NIST standards.

One of the fundamental problems in EC cryptography is the generation of cr yptographically secure ECs over prime fields, suitable for use in various cryptographic applications. A ty pical requirement of all such ap plications is that the order of the EC [22]. One of e ssential requirment for EC is its order (num ber of elem ents in the algebraic structure induced by the EC) possesses cert ain properties (e.g., robustnes s against known attacks [23], small prime factors [22, 24], etc), which gives rise to the problem of how such $\mathrm{E} \mathrm{C}$ can be generated. One of good decision of this tusk is curve of big prime order [24]. Also very important for this goal is avoidance curve of order $p+1$ because of it is tractable by to pairingbased att acks. As we have discussed before, supersi ngular elliptic curves ar e vulnerable to pairingbased attacks. Therefore we find a criterion of Edwards curve supersingularit $y$ [25]. The method of finding the order of a $n$ 
algebraic curve over a fi nite field $\mathrm{F}_{p^{n}}$ is now very relevant and is at the center of many mathematical studies in connection with the use of groups of points of cu rves of genus 1. In our article, this problem is solved.

Our algorithm has much less complexity for algebraic extensions with a la rge degree of finite fields. This is so because choosing suffi ciently large values $n$, we ontain $O\left(\log _{2}^{8} p^{n}\right)$ the value is much larger than $O\left(p \log _{2}^{2} p\right)$ for a fixed $\mathrm{v}$ alue $p$. The criterion of supersingularity of the Edw ards curves is found over $\mathrm{F}_{p^{n}}$. We additionall y propose a method for counting the points fr om Edwards curves and elliptic curves in response to an earlier paper by Schoof [8]. We consider the algebraic affine and projective Edw ards curves over a finite field. We not only find a s pecific set of coefficients with corresponding field characteristics for which supersingular, but we additionall $\mathrm{y}$ find a general formula by which one can determ ine whether a curve $E_{d}\left[\mathrm{~F}_{p}\right]$ is supersingular over this field o r not.

All proofs and anal ytical results belong $t \quad o$ Skuratovskii R. and computational examples, confirming statements, are made by Osadchyy V.

\section{Algebraic analyses of the curve and Curve Order Calculation Method}

We recall that the twisted Edwards curve with coefficients $a, d \in F_{p}{ }^{*}, d \neq 1, p \neq 2, a \neq d$, is the curve $E_{a, d}$ :

$$
a x^{2}+y^{2}=1+d x^{2} y^{2}, a, d \in F_{p}^{*}, a d(a-d) \neq 0,
$$

It should be noted that a twisted Edwards curve is called an Edwards curve when $a=1$. We denote by $E_{d}$ the Edwards curve with coefficient $d \in F_{p}{ }^{*}$ which is defined as $x^{2}+y^{2}=1+d x^{2} y^{2}$ over $\mathrm{F}_{p}$. The projective curve has form $F(x, y, z)=a x^{2} z^{2}+y^{2} z^{2}=z^{4}+d x^{2} y^{2}$. The special points are the infinitely distant points $(1,0,0)$ and $(0,1,0)$ and therefore we find its singularities at infinity in the corresponding affine components $A^{1}:=a z^{2}+y^{2} z^{2}=z^{4}+d y^{2}, A^{2}: a x^{2} z^{2}+z^{2}=z^{4}+d x^{2}$. These are simple singularities.

We describe the structure of the local ring at the point $p_{1}$ whose elements are quotients of functions with the form $F(x, y, z)=\frac{f(x, y, z)}{g(x, y, z)}$, where the denominator cannot take the value of 0 at the singular point $p_{1}$. In particular, we note that a loca 1 ring which has two singularities consists of functions with the denominators are not divisible by $(x-1)(y-1)$.

We denote by $\delta_{\mathrm{p}}=\operatorname{dim}^{\overline{\mathrm{O}}_{\mathrm{p}}} / \mathrm{O}_{p}$, where $\mathrm{O}_{\mathrm{p}}$ denotes the local ring at the singular point $\quad p$ which is generated by the relations of regu lar functions $\mathrm{O}_{\mathrm{p}}=\left\{\frac{f}{g}:(g,(x-1)(y-1))=1\right\}$ and $\overline{\mathrm{O}}_{\mathrm{p}}$ denotes the whole closure of the local ring at the singular point $p$.

We find that $\delta_{p}=\operatorname{dim}^{\overline{0}_{p}} / \mathrm{o}_{p}=1$ is the dim ension of the factor as a vector s pace. Because the basis of extension $\overline{\mathrm{O}}_{p} / \mathrm{O}_{p}$ consists of just one element at each distinct point, we obtain that $\delta_{p}=1$. We then calculate the genus of the curve ac cording to Fulton [4].

$\rho^{*}(C)=\rho_{\alpha}(C)-\sum_{p \in E} \delta_{p}=\frac{(n-1)(n-2)}{2}-\sum_{p \in E} \delta_{p}=3-2=1$, where $\rho_{\alpha}(C)$ denotes the arithmetic genus of the curve $C$ with parameter $n=\operatorname{deg}(C)=4$. It should be noted that the supersingular points were discovered in [10]. Recall the curve has a genus of 1 and as such it is kn own to be is omorphic to a flat cubic curve, however, the curve is i mportantly not elliptic because of its singularity in the projective part. Both the Edwards curve and the twisted Edwards curve are isomorphic to so me affine part of the elliptic curve. The Edwards curve after normaliz ation is precisely a curve in the Weierstrass normal form, which was proposed by Montgomery [1] and will be denoted by $E_{M}$. Koblitz [4,5] tells us that one can detect if a curve is supersingular using the search for the curve when that curve has the same number of points as its torsion curve. Also an elliptic curve $E$ over $F_{q}$ is called supersingular if for ev ery finite extension $F_{q^{r}}$ there are no points in the group $E\left(F_{q^{\prime}}\right)$ of order $p$ [17]. It is known [ 1] that the transition from an Edw ards curve to the relat ed torsion curve is determined by the reflection $(\bar{x}, \bar{y}) \mapsto(x, y)=\left(\bar{x}, \frac{1}{\bar{y}}\right)$.

We recall an im portant result from Vinogradov [13] which will act as criterion for supersingularity. Lemma 2.1. Let $k \in \mathrm{N}$ and $p \in \mathrm{P}$. Then

$\sum_{k=1}^{p-1} k^{n} \equiv \begin{cases}0(\bmod p), & n \nmid(p-1), \\ -1(\bmod p), & n \mid(p-1),\end{cases}$ 
where $n \mid(p-1)$ denotes that $n$ is divisible by $p-1$. The order of a curve is precisely the number of its affine points with a neutral ele ment, where the group operation is well defined. It is known that the order of $x^{2}+y^{2}=1+d x^{2} y^{2}$ coincides with the order of the curve $x^{2}+y^{2}=1+d^{-1} x^{2} y^{2}$ over $F_{p}$. We will now strengthen an existing result given in [10]. We denote the number of poin ts with a neutral element of an affine Edwards curve over the finite field $\mathrm{F}_{p}$ by $N_{d[p]}$ and the number of points on the projective curve over the same field by $\bar{N}_{d[p]}$.

Theorem 2.1. If $p \equiv 3(\bmod 4)$ is prime and the following condition of supersingularity

$$
\sum_{j=0}^{\frac{p-1}{2}}\left(C_{\frac{p-1}{2}}^{j}\right)^{2} d^{j} \equiv 0(\bmod p)
$$

is true th en the or ders of $\mathrm{t}$ he curves $x^{2}+y^{2}=1+d x^{2} y^{2}$ and $x^{2}+y^{2}=1+d^{-1} x^{2} y^{2}$ over $F_{p}$ are equal to $\quad N_{d[p]}=p+1, \quad$ when $\left(\frac{d}{p}\right)=-1, \quad$ and $N_{d[p]}=p-3$, when $\left(\frac{d}{p}\right)=1$.

Proof. Consider the curve $E_{d}$ :

$$
x^{2}+y^{2}=1+d x^{2} y^{2} .
$$

Transform it into the form $y^{2}\left(1-d x^{2} y^{2}\right)=1-x^{2}$, then we express $y^{2}$ by applying a rational transformation which lead us to the curve $y^{2}=\frac{1-x^{2}}{1-d x^{2} y^{2}}$.

For analysis we transform it into the curve

$$
y^{2}=\left(x^{2}-1\right)\left(d x^{2}-1\right)
$$

We denote the number of poin ts from an affine Edwards curve over the finite field $\mathrm{F}_{p}$ by $M_{d[p]}$. This curve (3) has $M_{d[p]}=N_{d[p]}+\left(\frac{d}{p}\right)+1$ points, which is precisely $\left(\frac{d}{p}\right)+1$ greater than the num ber of points of curve $E_{d}$. Note that $\left(\frac{d}{p}\right)$ denotes the Legendre Symbol. Let $a_{0}, a_{1}, \ldots, a_{2 p-2}$ be the coefficients of the poly nomial $a_{0}+a_{1} x+\ldots+a_{2 p-2} x^{2 p-2}$, which was obtained from $\left(x^{2}-1\right)^{\frac{p-1}{2}}\left(d x^{2}-1\right)^{\frac{p-1}{2}}$ after opening the bra ckets. Thus, summing over all $x$ yields

$$
\begin{aligned}
& M_{d[p]}=\sum_{x=0}^{p-1} 1+\left(\left(x^{2}-1\right)\left(d x^{2}-1\right)\right)^{\frac{p-1}{2}}=p+\sum_{x=0}^{p-1}\left(x^{2}-1\right)^{\frac{p-1}{2}} . \\
& \cdot\left(d x^{2}-1\right)^{\frac{p-1}{2}} \equiv \sum_{x=0}^{p-1}\left(x^{2}-1\right)^{\frac{p-1}{2}}\left(d x^{2}-1\right)^{\frac{p-1}{2}}(\bmod p) .
\end{aligned}
$$

By opening the brackets in $\left(x^{2}-1\right)^{\frac{p-1}{2}}\left(d x^{2}-1\right)^{\frac{p-1}{2}}$, we have $a_{2 p-2}=(-1)^{\frac{p-1}{2}} \cdot d^{\frac{p-1}{2}} \equiv\left(\frac{d}{p}\right)(\bmod p)$. So, using

Lemma 2.1 we have

$$
M_{d[p]} \equiv-\left(\frac{d}{p}\right)-a_{p-1}(\bmod p) .
$$

We need to prove $\mathrm{t}$ hat $M_{d[p]} \equiv 1(\bmod p)$ if $p \equiv 3(\bmod 8)$ and $M_{d[p]} \equiv-1(\bmod p)$. We therefore have to show that $\quad M_{d[p]} \equiv-\left(\frac{d}{p}\right)-a_{p-1}(\bmod p)$ for $p \equiv 3(\bmod 4) \quad$ if $\sum_{j=0}^{\frac{p-1}{2}}\left(C_{\frac{p-1}{2}}^{j}\right)^{2} d^{j} \equiv 0(\bmod p) . \quad$ If we prove that $a_{p-1} \equiv 0(\bmod p)$, then it will follow from (3). Let us determ ine $a_{p-1}$ according to $\mathrm{N}$ ewton's binomial formula: $a_{p-1}$ is equal to the coefficient at $x^{p-1}$ in $\mathrm{t}$ he polynomial, which is obtained as a

product

$$
\left(x^{2}-1\right)^{\frac{p-1}{2}}\left(d x^{2}-1\right)^{\frac{p-1}{2}} \text {. }
$$

So, $a_{p-1}=(-1)^{\frac{p-1}{2}} \sum_{j=0}^{\frac{p-1}{2}} d^{j}\left(C_{\frac{p-1}{2}}^{j}\right)^{2}$. Actually, the following equality holds:

$$
\begin{aligned}
& \sum_{j=0}^{\frac{p-1}{2}} d^{j}\left(C_{\frac{p-1}{2}}^{\frac{p-1}{2}-j}\right)(-1)^{\frac{p-1}{2}-\left(\frac{p-1}{2}-j\right)} \cdot d^{j}\left(C_{\frac{p-1}{2}}^{j}\right)^{2}(-1)^{\frac{p-1}{2}-j}= \\
& =(-1)^{\frac{p-1}{2}} \sum_{j=0}^{\frac{p-1}{2}} d^{j} C_{\frac{p-1}{2}}^{\frac{p-1}{2}} \cdot C_{\frac{p-1}{2}}^{j}=(-1)^{\frac{p-1}{2}} \sum_{j=0}^{\frac{p-1}{2}} d^{j}\left(C_{\frac{p-1}{2}}^{j} .\right.
\end{aligned}
$$

Since $a_{p-1}=-\sum_{j=0}^{\frac{p-1}{2}}\left(C_{\frac{p-1}{2}}^{j}\right)^{2} d^{j}$, then exact num ber of affine points on no $\mathrm{n}$ supersingular curv e (3) is the following $M_{d[p]} \equiv-a_{2 p-2}-a_{p-1}$ exactly:

$$
M_{d[p]} \equiv-\left(\frac{d}{p}\right)+\sum_{j=0}^{\frac{p-1}{2}}\left(C_{\frac{p-1}{2}}^{j}\right)^{2} d^{j}(\bmod p) .
$$

According to the condition of this theorem $a_{p-1}=0$, therefore $M_{d[p]} \equiv-a_{2 p-2}(\bmod p)$. Consequently, in the case when $p \equiv 3(\bmod 4)$, where $p$ is prime and 
$\sum_{j=0}^{\frac{p-1}{2}}\left(C_{\frac{p-1}{2}}^{j}\right)^{2} d^{j} \equiv 0(\bmod p), \quad$ the curve $\quad E_{d} \quad$ has

$\left.N_{d[p]}=p-\left(\frac{d}{p}\right)-\left(\frac{d}{p}\right)+1\right)=p-1-2\left(\frac{d}{p}\right)$

affine points and a group of points of the curve completed by singular points has $p+1$ points.

Exact number of the poi nts has upper boun $\mathrm{d}$ $2 p+1$ because for every $x \neq 0$ corresponds two valuations of $y$, but for $x=0$ we have only one solution $y=0$. Taking into account that $x \in F_{p}$ we have exactly $p$ values of $x$. Also there are 4 pairs $( \pm 1,0)$ and $(0, \pm 1)$ which are points of $E_{d}$ thus $N_{d[p]}>1$. Thus, $N_{d[p]}=p+1$. This com pletes the proof.

Corollary 2.1. The orders of the curves $x^{2}+y^{2}=1+d x^{2} y^{2}$ and $x^{2}+y^{2}=1+d^{-1} x^{2} y^{2}$ over $F_{p}$ are equal to $N_{d[p]}=p+1=\bar{N}_{d[p]}$, when $\left(\frac{d}{p}\right)=-1$, and $\quad N_{d[p]}=p-3=\bar{N}_{d[p]}-4, \quad$ when $\quad\left(\frac{d}{p}\right)=1 \quad$ iff $p \equiv 3(\bmod 4)$ is prime and $\sum_{j=0}^{\frac{p-1}{2}}\left(C_{\frac{p-1}{2}}^{j}\right)^{2} d^{j} \equiv 0(\bmod p)$. In more details conditions $N_{d[p]}=p-3=\bar{N}_{d[p]}-4$, when $\left(\frac{d}{p}\right)=1 \quad$ and $\quad N_{d[p]}=p+1=\bar{N}_{d[p]}, \quad$ when $\left(\frac{d}{p}\right)=-1$, imply (1), due to the formula of number of points (5) and deduced from (5) form ula (6) of affine points number of curve (2) $N_{d[p]}=p-\left(\frac{d}{p}\right)-\left(\left(\frac{d}{p}\right)+1\right)=p-1-2\left(\frac{d}{p}\right)$. Since all transformations in pro of of Theorem 2.1. were equivalent transitions then we obtain the proof of equivalence of conditions.

Theorem 2.2. If the coefficient $d=2$ or $d=2^{-1}$ and $p \equiv 3(\bmod 4)$ then $\sum_{j=0}^{\frac{p-1}{2}} d^{j}\left(C_{\frac{p-1}{d}}^{j}\right)^{2} \equiv 0(\bmod p)$ and $\bar{N}_{d[p]}=p+1$.

Proof. When $p \equiv 3(\bmod 4)$, we shall show that

$\sum_{j=0}^{\frac{p-1}{2}} d^{j}\left(C_{\frac{p-1}{d}}^{j}\right)^{2} \equiv 0(\bmod p)$. We multiply each binomial coefficient in this sum by $\left(\frac{p-1}{2}\right)$ ! to obtain after some algebraic manipulation

$\left(\frac{p-1}{2}\right) ! C_{\frac{p-1}{2}}^{j}=\frac{\left(\frac{p-1}{2}\right)\left(\frac{p-1}{2}-1\right) \cdots\left(\frac{p-1}{2}-j+1\right)\left(\frac{p-1}{2}\right) !}{1 \cdot 2 \cdots j}=$ $=\left(\frac{p-1}{2}\right)\left(\frac{p-1}{2}-1\right) \cdots\left(\frac{p-1}{2}-j+1\right)\left[\left(\frac{p-1}{2}\right)\left(\frac{p-1}{2}-1\right) \cdot \ldots\right.$ $\cdot(j+1)]$.

After applying the congr uence $\left(\frac{p-1}{2}-k\right)^{2} \equiv\left(\frac{p-1}{2}+1+k\right)^{2}(\bmod p)$ with $0 \leq k \leq \frac{p-1}{2}$ to the multipliers in previous parentheses, we obtain $\left[\left(\frac{p-1}{2}\right)\left(\frac{p-1}{2}-1\right) \cdots(j+1)\right]$.

It yields $\left(\frac{p-1}{2}\right)\left(\frac{p-1}{2}-1\right) \cdots\left(\frac{p-1}{2}-j+1\right)$.

$\left[\left(\frac{p-1}{2}+1\right) \cdots\left(\frac{p-1}{2}+\frac{p-1}{2}-j\right)\right](-1)^{\frac{p-1}{2}-j}$.

Thus, as a result of squaring, we have:

$\left(\left(\frac{p-1}{2}\right) ! C_{\frac{p-1}{2}}^{j}\right)^{2} \equiv\left(\frac{p-1}{2}-j+1\right)^{2}\left(\frac{p-1}{2}-j+2\right)^{2}$.

$\cdots(p-j-1)^{2}(\bmod p)$

It remains to prove that $\sum_{j=0}^{\frac{p-1}{2}}\left(C_{\frac{p-1}{2}}^{j}\right)^{2} 2^{j} \equiv 0(\bmod p)$ if $p \equiv 3(\bmod 4)$

Consider the auxillary

polynomial $P(t)=\left(\frac{p-1}{2} !\right)^{2} \sum_{j=0}^{\frac{p-1}{2}}\left(C_{\frac{p-1}{2}}^{j}\right)^{2} t^{j}$. We are going to show that $P(2)=0$ and the refore $a_{p-1} \equiv 0(\bmod p)$. Using (7) it can be shown that $a_{p-1}=P(t)=\left(\frac{p-1}{2} !\right)^{2} \sum_{j=0}^{\frac{p-1}{2}}\left(C_{\frac{p-1}{2}}^{j}\right)^{2} t^{j} \equiv \sum_{j=0}^{\frac{p-1}{2}}(k+1)^{2}$. $\cdot(k+2)^{2} \ldots\left(\frac{p-1}{2}+k\right)^{2} t^{k}(\bmod p)$

over $F_{p}$. We replace $d$ by $t$ in (1) such that we can research a more generalised problem. It should be noted tha $\mathrm{t} P(t)=\partial^{\frac{p-1}{2}}\left(\partial^{\frac{p-1}{2}}\left(Q(t) t^{\frac{p-1}{2}}\right) t^{\frac{p-1}{2}}\right)$ over $F_{p}$, where $Q(t)=t^{p-1}+\ldots+t+1$ and $\partial^{\frac{p-1}{2}}$ denotes the $\frac{p-1}{2}$-th derivative by $t$, where $t$ is new variable but not a coordinate of curve. Observe that $Q(t)=\frac{t^{p}-1}{t-1} \equiv \frac{(t-1)^{p}}{t-1} \equiv(t-1)^{p-1}(\bmod p) \quad$ and 
therefore the equality

$P(t)=\left(\left((t-1)^{p-1} t^{\frac{p-1}{2}}\right)^{\left(\frac{p-1}{2}\right)} t^{\frac{p-1}{2}}\right)^{\left(\frac{p-1}{2}\right)}$ holds over $\mathrm{F}_{p}$.

In order to si mplify notation we let $\theta=t-1$ and $R(\theta)=P(\theta+1)$. For the case $t=2$ we have $\theta=1$. Performing this substitution leads the pol ynomial $P(t)$ of 2 to $t$ he polynomial $R(t-1)$ of 1 . Takin $\mathrm{g}$ into account the linear nature of the substitution $\theta=t-1$, it can be seen that that derivation by $\theta$ and $t$ coincide. Derivat ion leads us to the transformation of polynomial $R(\theta)$ to form where it has the necess ary coefficient $a_{\mathrm{p}-1}$. Then $R(\theta)=P(\theta+1)=\partial^{\frac{p-1}{2}}\left(\partial^{\frac{p-1}{2}}\left(\theta^{p-1}(\theta+1)^{\frac{p-1}{2}}\right)(\theta+1)^{\frac{p-1}{2}}\right)=$ $=\partial^{\frac{p-1}{2}}\left(\frac{(p-1) !}{((p-1) / 2) !} \theta^{\frac{p-1}{2}}(\theta+1)^{\frac{p-1}{2}}\right)$.

In order to prove that $a_{p-1} \equiv 0(\bmod p)$, it is now sufficient to s how that $R(\theta)=0$ if $\theta=1$ over $F_{p}$. We obtain $R(1)=\frac{(p-1) !}{\left(\frac{p-1}{2}\right) !} \sum_{j=0}^{\frac{p-1}{2}} C_{\frac{p-1}{2}}^{j}(j+1) \cdots\left(j+\frac{p-1}{2}\right)$. We now will manipulate with the expression $\left(\frac{p-1}{2}-j+1\right)\left(\frac{p-1}{2}-j+2\right) \cdots\left(\frac{p-1}{2}-j+\frac{p-1}{2}\right)$.

order to ill ustrate the simplification we now consider the scena rio when $p=11$ and hence $\frac{p-1}{2}=5 . \quad$ The expression gets the $\mathrm{f}$ orm $(5-j+1)(5-j+2) \cdots(5-j+5)=(6-j)(7-j) \cdots(10-j) \equiv$ $\equiv((-5-j)(-4-j) \cdots(-1-j)) \equiv$ $\equiv(-1)^{5}((j+1)(j+2) \cdots(j+5))(\bmod 11)$.

Therefore, for a prime $p$, we can rewrite the expression $\left(\frac{p-1}{2}-j+1\right)\left(\frac{p-1}{2}-j+2\right) \cdots\left(\frac{p-1}{2}-j+\frac{p-1}{2}\right) \equiv$ $\equiv(-1)^{\frac{p-1}{2}}(j+1) \cdots\left(j+\frac{p-1}{2}\right)(\bmod p)$.

As a result, the sy mmetrical terms in (7) can be reduced yielding $a_{p-1} \equiv 0(\bmod p)$. It should be noted that $(-1)^{\frac{p-1}{2}}=-1$ since $p=M k+3$ and $\frac{p-1}{2}=2 k+1 . \quad$ Consequently, we have $P(2)=R(1)=0 \quad$ and henc $\quad$ e $\quad a_{p-1} \equiv 0(\bmod p) \quad$ as required. Thus, $\sum_{j=0}^{\frac{p-1}{2}}\left(C_{\frac{p-1}{2}}^{j}\right)^{2} \equiv 0(\bmod p)$, completing the proof of the of the theorem. The complexity of calculating of (1) is $O\left(p \log _{2}^{2} p\right)$ that will be prove $\mathrm{d}$ in Theorem 2.4.
Corollary 2.2. The curve $E_{d}$ is supersingular iff $E_{d^{-1}}$ is supersingular.

Proof. Let us recall the proved fact in Theorem 2.1 that $N_{d[p]} \equiv-a_{2 p-2}-a_{p-1} \equiv-\left(\frac{d}{p}\right)+\sum_{j=0}^{\frac{p-1}{2}}\left(C_{\frac{p-1}{2}}^{j}\right)^{2} d^{j}(\bmod p)$. Since $\left(C_{\frac{p-1}{2}}^{j}\right)^{2} d^{j} \equiv 0(\bmod p)$ by condition, and the congruence $\left(\frac{d}{p}\right) \equiv\left(\frac{d^{-1}}{p}\right)$ holds, then according to (6) the number of poi nts on $E_{d}$ is $N_{d[p]} \equiv-a_{2 p-2}-a_{p-1} \equiv-\left(\frac{d}{p}\right) \equiv\left(\frac{d}{p}\right)(\bmod p), \quad$ also $N_{d[p]} \equiv N_{d^{-1}[p]}$.

Corollary 2.3. If $p \equiv 3(\bmod 4)$, is pri me then $N_{d[p]}=p-1-2\left(\frac{d}{p}\right)+T$, where $T$ is such that $T \equiv \sum_{j=0}^{\frac{p-1}{2}}\left(C_{\frac{p-1}{2}}^{j}\right)^{2} d^{j} \bmod p$ and $T \leq 2 \sqrt{p}$.

Proof. Due to equality (5) and the bounds (8) as well as according to generalized Has se-Weil theorem $\left|N_{d[p]}-(p+1)-2\left(\frac{d}{p}\right)\right| \leq 2 g \sqrt{p}$, where $g$ is genus of curve, we obtain exact num ber $N_{d[p]}$. As we showed, $g=1$. From Theorem 2.1 as well as fro $\mathrm{m}$ Corollary $\quad 2.2$ we get, tha

$\sum_{j=0}^{\frac{p-1}{2}}\left(C_{\frac{p-1}{2}}^{j}\right)^{2} d^{j} \equiv-N_{d[p]}-(p+1)-2\left(\frac{d}{p}\right)$ so there exists $T \in Z$, such that $\quad T<2 \sqrt{p} \quad$ and $N_{d[p]}=p-1-2\left(\frac{d}{p}\right)+T$.

Example 2.1. If $p=13, d=2$ gives $N_{2[13]}=8$ and $p=13, d^{-1}=7$ gives that the number of points of $E_{7}$ is $N_{7[13]}=20$, which is in contradiction to that suggested by A. Bessalo $\mathrm{v}$ and $\mathrm{O}$. Thsigankova. Moreover, if $p \equiv 7(\bmod 8)$, then the order of torsion subgroup of curve is $N_{2}=N_{2^{-1}}=p-3$, which is clearly different to $p+1$ as suggested by $\mathrm{A}$. Bessalov and $\mathrm{O}$. Thsigankova.

For instance $\quad p=31$, then $N_{2[3]]}=N_{\left.2^{-1}[3]\right]}=28=31-3$, which is clearly not equal to $p+1$. If $p=7, d=2^{-1} \equiv(4 \bmod 7)$ then the curve $E_{2^{-1}}$ has four poi nts, namely $(0,1) ;(0,6) ;(1,0) ;(6,0)$, and the in case $p=7$ with 
$d=2(\bmod 7)$, the curve $E_{2^{-1}}$ also has four points : $(0,1) ;(0,6) ;(1,0) ;(6,0)$, demonstrating the order in this scenario is $p-3$.

The following theorem shows that the total number of affine points $u$ pon the Edw ards curves $E_{d}$ and $E_{d^{-1}}$ are equal under certain assu mptions.

This theorem additionally provides us with a formula for enumerating the number of affine points upon the birationally isomorphic Montgomery curve $N_{M}$.

Theorem 2.3. Let $d$ satisfy the condition of supersingularity (1). If $n \equiv 1(\bmod 2)$ and $p$ is prime, then $\bar{N}_{d\left[p^{n}\right]}=p^{n}+1$ and the order of curve is equal to $N_{d\left[p^{n}\right]}=p^{n}-1-2\left(\frac{d}{p}\right)$.

If $n \equiv 0(\bmod 2)$ and $p$ is prime, then the order of curve

$N_{d\left[p^{n}\right]}=p^{n}-3-2(-p)^{\frac{n}{2}}$, and the or der of projective curve is equal to $\bar{N}_{d\left[p^{n}\right]}=p^{n}+1-2(-p)^{\frac{n}{2}}$.

If $n \equiv 0(\bmod 2)$ and $p$ is prime, then the order of projective curve is equal to $\bar{N}_{d\left[p^{n}\right]}=p^{n}+1-2(-p)^{n / 2}$, and the order of affi ne curve $\mathrm{i} s$ equal to $N_{d\left[p^{n}\right]}=p^{n}+3-2(-p)^{n / 2}$.

Proof. We consider the extension of the base field $F_{p}$ to $F_{p^{n}}$ in order to determ ine the number of the points on the curve $x^{2}+y^{2}=1+d x^{2} y^{2}$. Let $P(x)$ denotes a pol ynomial with degree $m>2$ whose coefficients are from $\mathrm{F}_{p}$. To make the $\mathrm{p}$ roof, we take into account that it is known [12] that the number of solutions to $y^{2}=P(x)$ over $\mathrm{F}_{p^{n}}$ will have the form $p^{n}+1-\omega_{1}^{n}-\ldots-\omega_{m-1}^{n}$, where $\omega_{1}, \ldots, \omega_{m-1} \in \square$, $\left|\omega_{i}\right|=p^{\frac{1}{2}}$.

In case o $f$ our supersingular curve, if $n \equiv 1(\bmod 2)$ the num ber of points o $\mathrm{n}$ projective curve over $\mathrm{F}_{p^{n}}$ is deter mined by the expression $p^{n}+1-\omega_{1}^{n}-\omega_{2}^{n}$, where $\omega_{i}^{n} \in \square \quad$ and $\quad \omega_{1}=-\omega_{2}$, $\left|\omega_{i}\right|=\sqrt{p}$ that's why $\omega_{1}=i \sqrt{p}, \omega_{2}=-i \sqrt{p}$ with $i \in\{1,2\}$. In the general case, it is known [ $12,15,19]$ that $\left|\omega_{i}\right|=p^{\frac{1}{2}}$. The or der of the pr ojective curve is therefore $p^{n}+1$.

If $p \equiv 7(\bmod 8)$, then it is known from a result of

Skuratovskii [10] that $E_{d}$ has in its projective closure of the curve singular points w hich are not affine and therefore $N_{d[p]}=p^{n}-3$.

If $p \equiv 3(\bmod 8)$, then there are no singular points, hence $\bar{N}_{d[p]}=N_{d[p]}=p^{n}+1$. Consequently the number of points on the $\mathrm{E}$ dwards curve depends on $\left(\frac{d}{p}\right)$ and is equal to $N_{d[p]}=p^{n}-3$ if $p \equiv 7(\bmod 8)$ and $\quad N_{d[p]}=p^{n}+1 \quad$ if $p \equiv 3(\bmod 8) \quad$ where $n \equiv 1(\bmod 2)$. We note that this is because the transformation of (3) i n $E_{d}$ depends upon the denominator $\left(d x^{2}-1\right)$. If $n \equiv 1(\bmod 2)$ then, with respect to $t$ he sum of root of the $\mathrm{c}$ haracteristic equation for the Frobeniu s endomorphism $\omega_{1}^{n}+\omega_{2}^{n}$, which in this case have the same signs, we obtain that the number of points in the group of points of the curve is $p^{n}+1-\omega_{1}^{n}-\omega_{2}^{n}$ [19]. In more details $\omega_{1}, \omega_{2}$ are eigen values of Frobenius operator $F$ endomorphism on etale cohomology over the finite field $\mathrm{F}_{p^{n}}$, where $F$ acts of $H^{i}(X)$. The number of points, in general cas e, are determined by Lefshitz formula:

$$
\# X\left(\mathrm{~F}_{p^{n}}\right)=\sum(-1)^{i} \operatorname{tr}\left(\mathrm{F}^{n} \mid H^{i}(X)\right)
$$

where $\# X\left(\mathrm{~F}_{p^{n}}\right)$ is a num ber of points in the manifold $X$ over $\mathrm{F}_{p^{n}}, F^{n}$ is co mposition of the Frobenius operator. In our case, $E_{d}$ is considered as the manifold $X$ over $\mathrm{F}_{p^{n}}$.

For $n \equiv 0(\bmod 2)$ we a lways have, that every $d \in F_{p}$ is a quadratic residue in $F_{p^{n}}$. Consequently, because of $\left(\frac{d}{p}\right)=1$ four singula $\mathrm{r}$ points appe ar on the curve. Thus, the num ber of affine points is less by $4, \quad$ i.e. $N_{d\left[p^{n}\right]}=p^{n}-1-2\left(\frac{d}{p}\right)-2(-p)^{\frac{n}{2}}=p^{n}-3-2(-p)^{\frac{n}{2}}$.

Lemma 2.2. There exists birational iso morphism between $E_{d}$ and $E_{M}$, which is determ ined by correspondent mappings $x=\frac{1+u}{1-u}$ and $y=\frac{2 u}{v}$. Proof. To verify this statement in supersingular case we suppose that the curve $x^{2}+y^{2}=1+d x^{2} y^{2}$ contains $p-1-2\left(\frac{d}{p}\right)$ points $(x, y)$, with coordinates over prime field $\mathrm{F}_{p}$. Consider the transformation of the curve $x^{2}+y^{2}=1+d x^{2} y^{2}$ into the following form 
$y^{2}\left(d x^{2}-1\right)=x^{2}-1$. Make the substitutions $x=\frac{1+u}{1-u}$ and $y=\frac{2 u}{v}$. We will call the special points of this transformations the point in $\mathrm{w}$ hich these transformations or inverse transform ations are not determined. As a re sult the equation of curve the equation of the curve takes the form

$\frac{4 u^{2}}{v^{2}} \cdot \frac{(d-1) u^{2}+2(d+1) u+(d-1)}{(1-u)^{2}}=\frac{4 u}{(1-u)^{2}}$. Multipl y the equation of the curve by $\frac{v^{2}(1-u)^{2}}{4 u}$. As a result of the reduction, we obtain th e equation $v^{2}=(d-1) u^{3}+2(d+1) u^{2}+(d-1) u$. We an alyze what new solutions appeared in the resulting equation in comparing with $y^{2}\left(d x^{2}-1\right)=x^{2}-1$. First, there is an additional solution $(\mathrm{u}, \mathrm{v})=(0,0)$. Second, if $d$ is a quadratic residue by modulo $p$, then the following solutions appear: $\quad\left(u_{1}, v_{1}\right)=\left(\frac{-(d+1)-2 \sqrt{d}}{d-1}, 0\right)$, $\left(u_{2}, v_{2}\right)=\left(\frac{-(d+1)+2 \sqrt{d}}{d-1}, 0\right)$. If $\left(\frac{d}{p}\right)=-1$ then as it was shown above the order of $E_{d}$ is equal to $p+1$. Therefore, in cas e $\left(\frac{d}{p}\right)=-1$ order of $E_{d}$ appears one additional solution of from $(u, 0)$ more exact it is point with coordi nates $(0,0)$ also two points $((-1 ; 0),(1 ; 0))$ of $E_{d}$ have not images on $E_{M}$ in result of action of birational map on $E_{\mathrm{M}}$. Thus, in this case, number of affine points on $E_{M}$ is equal to $p+1-2+1=p$.

If $x=-1$ then equality $x=\frac{1+u}{1-u}$ transforms to form $-1+u=1+u$, or $-1=1$ that is $i$ mpossible for $p>2$. Therefore point $(-1,0)$ have not an image on $E_{M}$. Consider the ca se $x=1$. As a re sult of the substitutions $x=(1+u) /(1-u), y=2 u / v \quad$ we get the pair $(x, y)$ corresponding to the pair $(u, v)$ for which $v^{2}=(d-1) u^{3}+2(d+1) u^{2}+(d-1) u$.

If it occurs that $y=0$, then the preimage having coordinates $u=0$ and $v$ is not equal to 0 is suitable for the birational map $y=\frac{2 u}{v}$ which implies that $u=0$ and $v \neq 0$. But pair $(u, v)$ of such form do not satisfies the equation of obtained in result of mapping equation of Montgomery curve $v^{2}=(d-1) u^{3}+2(d+1) u^{2}+(d-1) u$. The table of correspondence between points is the following:

\begin{tabular}{|l|c|}
\hline Special points of $E_{M}$ & Special points of $E_{d}$ \\
\hline$(0 ; 0)$ & - \\
\hline$\left(\frac{-(d+1)-2 \sqrt{d}}{d-1}, 0\right)$ & - \\
\hline$\left(\frac{-(d+1)+2 \sqrt{d}}{d-1}, 0\right)$ & - \\
\hline$(1,-2 \sqrt{d})$ & - \\
\hline$(1,2 \sqrt{d})$ & - \\
\hline- & $(-1,0)$ \\
\hline- & $(1,0)$ \\
\hline
\end{tabular}

Table 1: Special points of birational maping.

The points $\left(\frac{-(d+1)-2 \sqrt{d}}{d-1}, 0\right),\left(\frac{-(d+1)+2 \sqrt{d}}{d-1}, 0\right)$, $(1,-2 \sqrt{d}),(1,2 \sqrt{d})$ exist on $E_{M}$ only when $\left(\frac{d}{p}\right)=1$. These points are element $\mathrm{s}$ of group which can be presented on Rie mann sphere over $F_{q}$. The points $(1,-2 \sqrt{d}),(1,2 \sqrt{d})(1,2 \sqrt{d})$ have not images on $E_{d}$ because of in denominator of transformations $x=\frac{1+u}{1-u}$ appears zero. By the same reason points $\left(\frac{-(d+1)-2 \sqrt{d}}{d-1}, 0\right), \quad\left(\frac{-(d+1)+2 \sqrt{d}}{d-1}, 0\right)$ have not an images on $E_{\mathrm{d}}$. If $\left(\frac{d}{p}\right)=1$ then as $\mathrm{i} \mathrm{t}$ was shown above the or der of $E_{d}$ is equal to $p-3$. Therefore order of $E_{M}$ is equal to $p$ because of 5 additional solutions of equation of $E_{M}$ appears but 2 points $((-1 ; 0),(1 ; 0))$ of $E_{d}$ have not images on $E_{M}$. These are 5 additional points ap pointed in tableau above. Also it exist $\mathrm{s}$ one infinit ely distant point on a $\mathrm{n}$ Montgomery curve. Thus, the order of $E_{M}$ is equal $p+1$ in this case as supersingular curve has.

The proof if complicated.

It should be noted that the supersingular curve $E_{d}$ is birationally equivalent to the supersingular elliptic curve which may be presented in Montgomery form $v^{2}=(d-1) u^{3}+2(d+1) u^{2}+(d-1) u$. As wel 1 as exceptional points [1] for the birational equivalence $(u, v) \mapsto(2 u / v,(u-1) /(u+1))=(x, y)$ are in one to one correspondence to the affine point of order 2 on $E_{d}$ and to the points in pro jective closure of $E_{d}$. Since the formula for number of affine points o $\mathrm{n}$ $E_{M}$ can b e applied to counting $N_{d[p]}$. In such way 
we apply this result $[7,12]$, to the ca se $y^{2}=P(x)$, where $\operatorname{deg} P(x)=m, \quad m=3$. The order $N_{M\left[p^{n}\right]}$ of the curve $E_{M}$ over $F_{p^{k}}$ can be evaluated due to Stepanov $[12,15]$. The $\mathrm{r}$ esearch tells us that th $\mathrm{e}$ order is $\bar{N}_{M\left[p^{n}\right]}=p^{n}+1-\omega_{1}^{n}-\omega_{2}^{n}$, where $\omega_{i}^{n} \in \square$ and $\omega_{1}^{n}=-\omega_{2}^{n}, \quad\left|\omega_{i}\right|=\sqrt{p}$ with $i \in\{1,2\}$. Therefore, we conclude when $n \equiv 1(\bmod 2)$, we know the order of Montgomery curve is precisely $N_{M\left[p^{n}\right]}=p^{n}+1$.

This result leads us to the conclusion that the number of solutions of $x^{2}+y^{2}=1+d x^{2} y^{2}$ as well as $v^{2}=(d-1) u^{3}+2(d+1) u^{2}+(d-1) u \quad$ over the finite field $\mathrm{F}_{p^{n}}$ are determined by the expression $p^{n}+1-\omega_{1}^{n}-\omega_{2}^{n}$ if $n \equiv 1(\bmod 2)$.

Example 2.2. The elliptic curve presented in the form of Mont gomery $E_{M}: v^{2}=u^{3}+6 u^{2}+u$ is birationally equivalent [ 1] to the curve $x^{2}+y^{2}=1+2 x^{2} y^{2}$ over the field $F_{p^{k}}$.

Corollary 2.4. If $d=2, n \equiv 1(\bmod 2) \quad$ and $p \equiv 3(\bmod 8)$, then the order of curve $E_{d}$ and order of the projective curv e are the following: $N_{d\left[p^{n}\right]}=p^{n}+1, \bar{N}_{d\left[p^{n}\right]}=p^{n}+1$.

If $d=2, n \equiv 1(\bmod 2)$ and $p \equiv 7(\bmod 8)$, then the number of points of projective curve is

$$
\bar{N}_{d\left[p^{n}\right]}=p^{n}+1,
$$

and the number of points on affine curve $E_{d}$ is also

$$
\bar{N}_{d\left[p^{n}\right]}=p^{n}-3 .
$$

In case $d=2, \quad n \equiv 0(\bmod 2), \quad p \equiv 3(\bmod 4)$, the general formula of the curves order is

$$
N_{d\left[p^{n}[\right.}=p^{n}-3-2(-p)^{\frac{n}{2}} .
$$

The general formula for $n \equiv 0(\bmod 2)$ and $d=2$ for the number of points on projective curve for the supersingular case is

$$
\bar{N}_{d\left[p^{n}\right]}=p^{n}+1-2(-p)^{\frac{n}{2}} .
$$

Proof. We denote by $N_{M\left[p^{n}\right]}$ the order of the curve $E_{M}$ over $F_{p^{n}}$. The order $N_{M\left[p^{n}\right]}$ of $E_{M}$ over $F_{p^{n}}$ can be evaluated [6] as $N_{M\left[p^{n}\right]}=p^{n}+1-\omega_{1}^{n}-\omega_{2}^{n}$, where $\omega_{i}^{n} \in \mathrm{C}$ and $\omega_{1}^{n}=-\omega_{2}^{n},\left|\omega_{i}\right|=\sqrt{p}$ with $i \in\{1,2\}$. For the finite alg ebraic extension of degree $n$, we will consider $\quad p^{n}-\omega_{1}^{n}-\omega_{2}^{n}=p^{n} \quad$ if $\quad n \equiv 1(\bmod 2)$. Therefore, for $n \equiv 1(\bmod 2)$, the o rder of th $\mathrm{e}$ Montgomery curve is precisely given by
$N_{M\left[p^{n}\right]}=p^{n}+1$. Here's one infinitely remote point as a neutral element of the group of points of the curve. Considering now an elliptic curve, we have $\omega_{1}=\bar{\omega}_{2}$ by [5], which leads to $\omega_{1}+\omega_{2}=0$. For $n=1$, it is clear that $N_{M}=p$. When $n$ is odd, we have $\omega_{1}^{n}+\omega_{2}^{n}=0$ and therefore $N_{M, n}=p^{n}+1$. Because $n$ is even by initial assu mption, we shal 1 show that $N_{M\left[p^{n}\right]}=p^{n}+1-2(-p)^{\frac{n}{2}}$ holds as required.

Note that for $n=2$ we can express the number as $\bar{N}_{d\left[p^{2}\right]}=p^{2}+1+2 p=(p+1)^{2} \quad$ with respect to Lagrange theorem have to be divisibl e on $\bar{N}_{d[p]}$. Because a group of $E_{d}\left(F_{p^{2}}\right)$ over square exten sion of $F_{p}$ contains the group $E_{d}\left(\mathrm{~F}_{p}\right)$ as a proper subgroup. In fact, according to Theorem 1 the order $E_{d}\left(\mathrm{~F}_{p}\right)$ is $p+1$ therefore divisibility of orde $\mathrm{r}$ $E_{d}\left(\mathrm{~F}_{p^{2}}\right)$ holds because in our case $p=7$ thus $\bar{N}_{E_{d}}=8^{2}$ and $p+1=8=N_{d[7]}[16]$. The following two examples exemplify Corollary 2.4 .

Example 2.3. If $p \equiv 3(\bmod 8)$ and $n=2 k$ then we have when $d=2, n=2, p=3$ that the num ber of affine points equals to

$N_{2[3]}=p^{n}-3-2(-p)^{\frac{n}{2}}=3^{2}-3-2 \cdot(-3)=12$,

and the $\mathrm{n}$ umber of projective points is equal to $\bar{N}_{2[3]}=p^{n}+1-2(-p)^{\frac{n}{2}}=3^{2}+1-2 \cdot(-3)=16$.

Example 2.4. If $p \equiv 7(\bmod 8)$ and $n=2 k$ then we have when $d=2, n=2, p=7$ that the num ber of affine points equals to $N_{2[7]}=p^{n}-3-2(-p)^{\frac{n}{2}}=7^{2}-3-2 \cdot(-7)=60$, and the number of projective poi nts is equal $\mathrm{t} \quad \mathrm{O}$ $\bar{N}_{2[7]}=p^{n}+1-2(-p)^{\frac{n}{2}}=7^{2}+1-2 \cdot(-7)=64$.

The group of points of the supersingular curve $E_{d}$ contains $p-1-2\left(\frac{d}{p}\right)$ affine points a nd the affine singular points whose number is $2\left(\frac{d}{p}\right)+2$.

The singular points were discovered in [ 10] and hence if the curve is free of singular points then the group order is $p+1$.

Example 2.5. The number of curve points over finite field when $d=2$ and $p=31$ is equal to $N_{2[31]}=N_{2^{-1}[31]}=p-3=28$. 
Theorem 2.4. The order of Edwards curve over $F_{p}$ is congruent to

$$
\begin{aligned}
& \bar{N}_{d[p]} \equiv\left(p-1-2\left(\frac{d}{p}\right)+(-1)^{\frac{p+1}{2}} \sum_{j=0}^{\frac{p-1}{2}}\left(C_{\frac{p-1}{2}}^{j}\right)^{2} d^{j}\right) \equiv \\
& \equiv\left((-1)^{\frac{p+1}{2}} \sum_{j=0}^{\frac{p-1}{2}}\left(C_{\frac{p-1}{2}}^{j}\right)^{2} d^{j}-1-2\left(\frac{d}{p}\right)(\bmod p) .\right.
\end{aligned}
$$

The true value of $\bar{N}_{d[p]}$ lies in $[4 ; 2 p]$ and is even.

Proof. This result follows fro $\mathrm{m}$ the num ber of solutions of the equation $y^{2}=\left(x^{2}-1\right)\left(d x^{2}-1\right)$ over $F_{p}$

$$
\begin{aligned}
& \left.\left.\sum_{x=0}^{p-1}\left(\frac{\left(x^{2}-1\right)\left(d x^{2}-1\right)}{p}\right)+1\right) \equiv \sum_{x=0}^{p-1}\left(\frac{\left(x^{2}-1\right)\left(d x^{2}-1\right)}{p}\right)\right)+p \equiv \\
& \equiv\left(\sum_{j=0}^{\frac{p-1}{2}}\left(x^{2}-1\right)^{\frac{p-1}{2}}\left(d x^{2}-1\right)^{\frac{p-1}{2}}\right)(\bmod p) \equiv \\
& \equiv\left((-1)^{\frac{p+1}{2}} \sum_{j=0}^{\frac{p-1}{2}}\left(C_{\frac{p-1}{2}}^{j}\right)^{2} d^{j}-\left(\frac{d}{p}\right)\right)(\bmod p) .
\end{aligned}
$$

The quantity of solutions for $x^{2}+y^{2}=1+d x^{2} y^{2}$ differs from the quantity of $y^{2}=\left(d x^{2}-1\right)\left(x^{2}-1\right)$ by $\left(\frac{d}{p}\right)+1$ due to new solutions in the from $(\sqrt{d}, 0),(-\sqrt{d}, 0)$. So this quantity is such

$$
\begin{aligned}
& \left.\sum_{x=0}^{p-1}\left(\frac{\left(x^{2}-1\right)\left(d x^{2}-1\right)}{p}\right)+1\right)-\left(\left(\frac{d}{p}\right)+1\right) \equiv \\
& \left.\sum_{x=0}^{p-1}\left(\frac{\left(x^{2}-1\right)\left(d x^{2}-1\right)}{p}\right)\right)+p-\left(\left(\frac{d}{p}\right)-1\right) \equiv \\
& \equiv\left(\sum_{j=0}^{\frac{p-1}{2}}\left(x^{2}-1\right)^{\frac{p-1}{2}}\left(d x^{2}-1\right)^{\frac{p-1}{2}}-\left(\frac{d}{p}\right)+1\right)(\bmod p) \equiv \\
& \equiv(-1)^{\frac{p+1}{2}} \sum_{j=0}^{\frac{p-1}{2}}\left(C_{\frac{p-1}{2}}^{j}\right)^{2} d^{j}-\left(2\left(\frac{d}{p}\right)+1\right)(\bmod p) .
\end{aligned}
$$

According to Lemma 1 the last sum $\left(\sum_{j=0}^{\frac{p-1}{2}}\left(x^{2}-1\right)^{\frac{p-1}{2}}\left(d x^{2}-1\right)^{\frac{p-1}{2}}\right)(\bmod p) \quad$ is congruent to $-a_{p-1}-a_{2 p-2}(\bmod p)$, where $a_{i}$ are the coefficients from presentation

$$
\left(x^{2}-1\right)^{\frac{p-1}{2}}\left(d x^{2}-1\right)^{\frac{p-1}{2}}=a_{0}+a_{1} x+\ldots+a_{2 p-2} x^{2 p-2} .
$$

Last presentation was obtained transformation

$$
\begin{aligned}
& \left(x^{2}-1\right)^{\frac{p-1}{2}}\left(d x^{2}-1\right)^{\frac{p-1}{2}}=\left(\sum_{x=0}^{p-1} C_{\frac{p-1}{2}}^{k} x^{2 k}(-1)^{\frac{p-1}{2}-k}\right) \\
& \left(\sum_{x=0}^{p-1} C_{\frac{p-1}{2}}^{j} d^{j} x^{2 j}(-1)^{\frac{p-1}{2}-j}\right) .
\end{aligned}
$$

Therefore $a_{2 p-2}$ is equal to $d^{\frac{p-1}{2}} \equiv\left(\frac{d}{p}\right)(\bmod p)$ and $a_{p-1}=\sum_{j=0}^{\frac{p-1}{2}}\left(C_{\frac{p-1}{2}}^{j}\right)^{2} d^{j}(-1)^{\frac{p-1}{2}}$.

According to Newton's binom ial formula $a_{p-1}$ equal to the coefficient at $x^{p-1}$ in the product of two brackets and when substituting this $d$ instead of 2 is such

$$
(-1)^{\frac{p-1}{2}} \sum_{j=0}^{\frac{p-1}{2}} d^{j}\left(C_{\frac{p-1}{2}}^{j}\right)^{2}
$$

that is, it has the form o f the poly nomial with inverse order of coefficients. Indeed, we have equality

$$
\begin{aligned}
& \sum_{j=0}^{\frac{p-1}{2}} d^{j}\left(C_{\frac{p-1}{2}}^{\frac{p-1}{2}-j}\right)(-1)^{\frac{p-1}{2}-\left(\frac{p-1}{2}-j\right)} \cdot\left(C_{\frac{p-1}{2}}^{j}\right)^{2}(-1)^{\frac{p-1}{2}-j}= \\
& =(-1)^{\frac{p-1}{2}} \sum_{j=0}^{\frac{p-1}{2}} d^{j} C_{\frac{p-1}{2}}^{\frac{p-1}{2}-j} \cdot C_{\frac{p-1}{2}}^{j}=(-1)^{\frac{p-1}{2}} \sum_{j=0}^{\frac{p-1}{2}} d^{j}\left(C_{\frac{p-1}{2}}^{j}\right)^{2} .
\end{aligned}
$$

In form of a su $\mathrm{m}$ it is the following

$$
\begin{aligned}
& \sum_{j=0}^{\frac{p-1}{2}} 2^{j}\left(C_{\frac{p-1}{2}}^{\frac{p-1}{2}-j}\right)(-1)^{\frac{p-1}{2}-\left(\frac{p-1}{2}-j\right)} \cdot 2^{j}\left(C_{\frac{p-1}{2}}^{j}\right)^{2}(-1)^{\frac{p-1}{2}-j}= \\
& =(-1)^{\frac{p-1}{2}} \sum_{j=0}^{\frac{p-1}{2}} 2^{j} C_{\frac{p-1}{2}}^{\frac{p-1}{2}-j} \cdot C_{\frac{p-1}{2}}^{j}=(-1)^{\frac{p-1}{2}} \sum_{j=0}^{\frac{p-1}{2}} 2^{j}\left(C_{\frac{p-1}{2}}^{j} .\right.
\end{aligned}
$$

over $\mathrm{F}_{p}$ equals to $p-1-2\left(\frac{d}{p}\right)+\left(1+\left(\frac{d}{p}\right)\right)=p-\left(\frac{d}{p}\right)$ and differs fro $m$ the quantity of solutions of $x^{2}+y^{2}=1+d x^{2} y^{2}$ by $\left(\frac{d}{p}\right)+1$ due to new solutions of $y^{2}=\left(d x^{2}-1\right)\left(x^{2}-1\right)$. Thus, in general c ase if $a_{p-1}=\sum_{j=0}^{\frac{p-1}{2}}\left(C_{\frac{p-1}{2}}^{j}\right)^{2} d^{j}(-1)^{\frac{p-1}{2}} \neq 0 \quad$ we have $N_{E_{d}}=\left(p-\left(\frac{d}{p}\right)-\left(\left(\frac{d}{p}\right)+1\right)-(-1)^{\frac{p-1}{2}} \sum_{j=0}^{\frac{p-1}{2}}\left(C_{\frac{p-1}{2}}^{\frac{p-1}{2}-j} C_{\frac{p-1}{2}}^{j}\right)^{2} d^{j}\right) \equiv$ $\equiv\left(p-1-(-1)^{\frac{p-1}{2}} \sum_{j=0}^{\frac{p-1}{2}}\left(C_{\frac{p-1}{2}}^{j}\right)^{2} d^{j}-2\left(\frac{d}{p}\right)\right) \equiv$ $\equiv\left((-1)^{\frac{p+1}{2}} \sum_{j=0}^{\frac{p-1}{2}}\left(C_{\frac{p-1}{2}}^{j}\right)^{2} d^{j}-1-2\left(\frac{d}{p}\right)\right)(\bmod p)$.

The exact order is not les $\mathrm{s}$ than 4 beca use cofactor of this curve is 4 . To determine the order is uniquely 
enough to take into acco unt that $p$ and $2 p$ have different parity. Taking into account that the order is even we chose a term $p$ or $2 p$, for the sum which define the order.

Let us analyze the complexity of calculating the value of $\sum_{j=0}^{\frac{p-1}{2}}\left(C_{\frac{p-1}{2}}^{j}\right)^{2} d^{j}$. B inomial coefficients of the form $C_{\frac{p-1}{2}}^{l}$ we calculate recursively having $C_{\frac{p-1}{2}}^{l}$ we get $C_{\frac{p-1}{2}}^{l+1}$. Such a transformation can be done by one multiplication of one division. But division can be avoided by applying the Legendre formula to count the number of occurrences of all prime factors from 2 to $(p-1): 2$. In $\mathrm{b}$ oth cases, the co mplexity of calculating all the coefficients from the sum (3) is equal to $O\left(\frac{p-1}{2} \log _{2}^{2} p\right)$. Squaring the calculated binomial coefficient $C_{\frac{p-1}{2}}^{j}$ also does not exceed $O\left(\log _{2}^{2} p\right)$. Calculate all values of $\quad d^{j} \bmod p$ optimally applying recursive multiplication $d^{j-1}$ on $d$ for this we use the Ka ratsuba multiplication method requiring $O\left(\log _{2}^{\log _{2} 3} p\right)$, than apply the Barrett method of modular multip lication. Therefore, the com plexity of computing th $\mathrm{e}$ entire tuple of degrees $d^{j}, j=1, \ldots, n$ is $O\left(\frac{p-1}{2} \log _{2}^{\log _{2} 3} p\right) . \quad \mathrm{T} \quad$ otally we obtai $\mathrm{n}$ $O\left(\frac{p-1}{2} \log _{2}^{2} p\right)$.

Theorem 2.6. If $\left(\frac{d}{p}\right)=1$, then the orders of the curves $E_{d}$ and $E_{d^{-1}}$, satisfies to the following relation $\left|E_{d}\right|=\left|E_{d^{-1}}\right|$.

If $\left(\frac{d}{p}\right)=-1$, then $E_{d}$ and $E_{d^{-1}}$ are pair of twisted curves i.e. orders of curves $E_{d}$ and $E_{d^{-1}}$ satisfies to the following relation of duality

$$
\left|E_{d}\right|+\left|E_{d^{-1}}\right|=2 p+2
$$

Let the curve be defined $\mathrm{b}$ $x^{2}+y^{2}=1+d x^{2} y^{2}(\bmod p)$, then we can express $y^{2}$ in such way:

$$
y^{2} \equiv \frac{x^{2}-1}{d x^{2}-1}(\bmod p .)
$$

For $x^{2}+y^{2}=1+d^{-1} x^{2} y^{2}(\bmod p)$ we could obtai $\mathrm{n}$ that

$$
y^{2} \equiv \frac{x^{2}-1}{d^{-1} x^{2}-1}(\bmod p)
$$

If $\left(\frac{d}{p}\right)=1$, then for the fixed $x_{0}$ a quantity of $y$ over $\mathrm{F}_{p}$ can becalcul ated by the for mula $\left(\frac{\frac{x^{2}-1}{d^{-1} x^{2}-1}}{p}\right)+1$ for $x$ such that $d^{-1} x^{2}+1 \equiv 0(\bmod p)$. For solution $\left(x_{0}, y_{0}\right)$ to $(10)$, we have the equality $y_{0}^{2} \equiv \frac{x_{0}^{2}-1}{d x_{0}^{2}-1}(\bmod p) \quad$ and we express $y_{0}^{2} \equiv \frac{1-\frac{1}{x_{0}^{2}}}{1-\frac{1}{d x_{0}^{2}}} d^{-1} \equiv \frac{\left(\frac{1}{x_{0}}\right)^{2}-1}{\frac{1}{d}\left(\frac{1}{x_{0}}\right)^{2}-1} d^{-1} \equiv \frac{\left(\frac{1}{x_{0}}\right)^{2}-}{d^{-1}\left(\frac{1}{x_{0}}\right)^{2}-1} d^{-1}$.

Observe that

$$
y^{2}=\frac{x^{2}-1}{d^{-1} x^{2}-1}=\frac{1-x^{2}}{1-d^{-1} x^{2}}=\frac{\left(\frac{1}{x^{2}}-1\right)}{\left(\left(\frac{d}{x^{2}}\right)-1\right)} d .
$$

Thus, if $\left(x_{0}, y_{0}\right)$ is solution of (2), then $\left(\frac{1}{x_{0}}, \frac{y_{0}}{\sqrt{d}}\right)$ is a solution to (10) because last transformations determines that $\frac{\mathrm{y}_{0}^{2}}{d} \equiv \frac{d^{-1}\left(\frac{1}{x_{0}}\right)^{2}-1}{\left(\frac{1}{x_{0}}\right)^{2}-1} \operatorname{modp} . \quad$ Therefore last transformations $\left(x_{0}, y_{0}\right) \rightarrow\left(\frac{1}{x_{0}}, \frac{y_{0}}{\sqrt{d}}\right)=(x, y)$ determines isomorphism and bijection.

In case $\left(\frac{d}{p}\right)=-1$, then every $x \in \mathrm{F}_{p}$ is such that $d x^{2}-1 \neq 0$ and $d^{-1} x^{2}-1 \neq 0$. If $x_{0} \neq 0$, then $x_{0}$ generate 2 solutions of (2) iff $x_{0}^{-1}$ gives 0 solution $\mathrm{s}$ of (10) because of (11) yields the following relation

$$
\left(\frac{\frac{x^{2}-1}{d^{-1} x^{2}-1}}{p}\right)=\left(\frac{\frac{x^{-2}-1}{d x^{-2}-1}}{p}\right)\left(\frac{d}{p}\right)=-\left(\frac{\frac{x^{-2}-1}{d x^{-2}-1}}{p}\right) .
$$

Analogous reasons give us that $x_{0}$ give exactly one solution of (2) iff $x_{0}^{-1}$ gives 1 solutions of (10). Consider the set $x \in\{1,2, \ldots, p-1\}$ we obtain that the total amount of solution $\mathrm{s}$ of form $\left(x_{0}^{-1}, y_{0}\right)$ that represent point of (2) and pairs of form $\left(x_{0}, y_{0}\right)$ that 
represent point of curve (10) is $2 p-2$. Also we have two solutions of (2) of form $(0,1)$ and $(0,-1)$ and two solutions of (10) that has form $(0,1)$ and $(0,-1)$. The proof is fully completed.

Example 2.6. The number of points of $E_{d}$ over $F_{p}$ for $p=13$ and $d=2$ is given by $N_{2[13]}=8$. In the case when $p=13$ and $d^{-1}=7$ we have that the number of points of $E_{7}$ is $N_{7[13]}=20$. Therefore, we have that the sum of orders for these curve is equal to $28=2 \cdot 13+2$ which confirms our theorem. The set of points over $F_{13}$ when $d=2$ are precisely $\{(0,1) ;(0,12) ;(1,0) ;(4,4) ;(4,9) ;(9,4) ;(9,9) ;(12,0)\}$, while for $d=7$, we have the set $\{(0,1) ;(0,12) ;(1,0)$; $(2,4) ;(2,9) ;(4,2) ;(4,11) ;(5,6) ;(5,7) ;(6,5) ;(6,8) ;(7,5)$; $(7,8) ;(8,6) ;(8,7) ;(9,2) ;(9,11) ;(11,4) ;(11,9) ;(12,0)\}$.

Example 2.7. If $p=7$ and $d=2^{-1} \equiv 4(\bmod 7)$, then we have $\left(\frac{d}{p}\right)=1$ and the curve $E_{2^{-1}}$ has four points which are $(0,1) ;(0,6) ;(1,0) ;(6,0)$. and the in case $p=7$ for $d=2(\bmod 7)$, the curve $E_{2^{-1}}$ also has four points which are $(0,1) ;(0,6) ;(1,0) ;(6,0)$.

Definition 2.1. We call the embedding degree a minimal power $k$ of a finite field extension such that the group of points of the curve can be embedded in the multiplicative group of $\mathrm{F}_{p^{k}}$.

Let us obtain conditions of em bedding [14] for the grou $\mathrm{p}$ of supersingular curves $E_{d}\left[\mathrm{~F}_{p}\right]$ of order $p$ in the multiplicative group of field $\mathrm{F}_{p^{k}}$ whose embedding degree is $k=12$ [14]. We now utilise the Zsigmondy theorem which implies that a suitable characteristic of field $\mathrm{F}_{p}$ is an arbitrary prime $p$ which do no $\mathrm{t}$ divide 12 and satisfies the condition $q \mid \mathrm{P}_{12}(p)$, where $\mathrm{P}_{12}(x)$ is the cy clotomic polynomial. This $p$ will satisfy the necessary conditions $\left(x^{n}-1\right) \mid p$ for an arbitrary $n=1, \ldots, 11$.

Proposition 2.1 The degree of embedding for the group of a supersingular curve $E_{d}$ is equal to 2.

Proof. The o rder of the group of a supersingular curve $E_{d}$ is equal to $p^{k}+1$. It should be observed that $p^{k}+1$ divides $p^{2 k}-1$, but $p^{k}+1$ does not divide expressions of the form $p^{2 l}-1$ with $l<k$. This division does not wo rk for smaller values of $l$ due to the decomposition of the expression $p^{2 k}-1=\left(p^{k}-1\right)\left(p^{k}+1\right)$. Therefore, we can use the definition to conclude that the degree of embedding must be 2 , confirming the proposition.
Consider $\mathrm{E}_{2}$ over $\mathrm{F}_{p^{2}}$, for instance we assume $p=3$. We define $\mathrm{F}_{9}$ as $\mathrm{F}_{3}(\alpha)$, where $\alpha$ is a root of $x^{2}+1=0$ over $\mathrm{F}_{9}$. Therefore elements of $\mathrm{F}_{9}$ have form: $a+b \alpha$, where $a, b \in \mathrm{F}_{3}$. So we assume that $x \in\{ \pm(\alpha+1), \pm(\alpha-1), \pm \alpha\} \quad$ and check its belonging to $\mathrm{E}_{2}$. For instance if $x= \pm(\alpha+1)$ then $x^{2}=\alpha^{2}+2 \alpha+1=2 \alpha=-\alpha . \quad$ Also in this case $y^{2}=\frac{2 \alpha-1}{\alpha-1}=\frac{(2 \alpha-1)(\alpha+1)}{(\alpha-1)(\alpha+1)}=\frac{(2 \alpha-1)(\alpha+1)}{(\alpha-1)(\alpha+1)}=\frac{\alpha}{-2}=\alpha$. Therefore the correspondent second coordinate is $y= \pm(\alpha-1)$. The si milar computations lead us to full the following list of curves points.

\begin{tabular}{|l|l|l|l|l|}
\hline$x$ & \pm 1 & 0 & $\pm(\alpha+1)$ & $\pm(\alpha-1)$ \\
\hline$y$ & 0 & \pm 1 & $\pm(\alpha-1)$ & $\pm(\alpha+1)$ \\
\hline
\end{tabular}

Table 2: Points of Edwards curve over square extension.

The total amount is 12 affine poin ts that confirms Corollary 2.4. and Theore $\mathrm{m}$ 2.3. because of $p^{n}-3-2(-p)^{\frac{n}{2}}=3^{2}-3-2(-3)=12$.

\section{Conclusion}

The new effective algorithm for the elli ptic and Edwards curves order curve counting was founded. The criterion for supersingularit $\mathrm{y}$ of $\mathrm{t}$ hese curves was additionally obtained.

\section{References:}

[1] Daniel J. Be rnstein, Peter Birkner, Marc Joy e, Tanja Lange, and Christ iane Peters. Twisted edwards curves. Progress in Cryptology -AFRICACRYPT 2008 (6,5);(6,8);(7,5);, pp. 389--405, Berlin, Heidelberg, 2008.

[2] Don Johnson, Alfred Menezes, Scott Vanstone. The Elliptic Curve Digital Signature Algorithm. International Journal of Information Security volume 1, Springer, 2014, pp. 36-63.

[3] Harold Edwards. A normal form fo $r$ elliptic curves. Bulletin of the American mathematical society, 44(3):393--422, 2007.

[4] William Fulton. Algebraic curves. An Introduction to Algebraic Geometry. AddisonWesley, 3 edition, 2008.L.

[5] Neal Koblitz. Elliptic curve cryptos ystems. Mathematics of computation, 48(177):203--209, 1987.

[6] Rudolf Lidl and Harald Niederreiter. Introduction to $\mathrm{Fi}$ nite Fields and $\mathrm{t}$ heir Applications. Cambridge university press, 1994.

[7] Peter L Montgomery. Speeding the pollard and elliptic curve methods of factorization. Mathematics of computation, 48(177):243--264, 1987.

[8] René Schoof. Counting points on elliptic curves over finite fields. Journal de théorie des nombres de Bordeaux, 7(1):219--254, 1995. 
[9] Ruslan Viacheslavovich Skuratovskii. The order of projective edwards curve ove $r$ an $d$ embedding degree of this curve in finite field. In Cait 2018, pp. 75 -- 80, 2018.

[10] Ruslan Viacheslavovich Skur atovskii. Supersingularity of elliptic curves. Research in Mathematics and Mechanics, 31(1):17--26, 2018.

[11] Ruslan Viacheslavovich Skur atovskii. Employment of $\mathrm{m}$ inimal generating sets and structure of sy low 2-subgroups alternating groups in block ciphers. In Advances in Computer Communication and Computational Sciences, pages 351--364. Springer, 2019.

[12] Serge Aleksandrovich Stepanov. Arifmetika algebraicheskikh krivykh (in Russian). Nauka, Glav. red. fiziko-matematichesko lit-ry, 1991.

[13] Ivan Matveevich Vinogradov. Elements of number theory. Courier Dover Publ ications, 2016.

[14] Paulo S. L. M. Barreto and Michael Naehrig. Pairing-friendly elliptic curves of prime order. Springer, Selected Areas in Cryptography, pages 319--331, Berlin, Heidelberg, 2006.

[15] N.M. Glazunov, Skobelev S.P. Manifolds over the rings. IAMM National Academy of Sciences of Ukraine, Donetsk, 2011.323 p.

[16] P.D Varbanec, P Zarzycki. Divisors of the Gaussian integers in an ar ithmetic progression. Journal of Number Theory. Volume 33, Issue 2, October 1989, Pages 152-169

[17] Silverman, Joseph, H.; The Arithmetic of Elliptic Curves, Graduate Texts in Mathematics, 106, Springer-Verlag, 1986.
[18] R. V. Skuratovskii, Aled William s (2019) "A solution of the inverse problem to doubling of twisted Edwards curve poi nt over finite field", Processing, transmission and security of information - 2019 vol. 2,

[19] Deligne, Pierre. La conjecture de Weil, Publications Mathematiques de l'IHEŚ. 1974. Vol. 43. pp. 273-307.IEEE

[20] R. V. Skura tovskii, Employment of Minimal Generating Sets and Structure of S ylow 2Subgroups Alternating Groups in Block Ciphers. Springer, Advances in Computer Communication and Computational Sciences, 2019, pp. 351-364.

[21] R. Skuratovskii, The Derived Subgr oups of Sylow 2-Subgroups of the Alternating Group and Commutator Width of Wreath Product of Groups. Mathematics, Basel, Switzerland, (2020) № 8(4), pp. 1-19.

[22] Craig Costello, Benjam in Smith Montgom ery curves and their arit hmetic. Journal of Cryptographic Engineering volume 8 no.3, pp. 227-240 (2018).

[23] Andrea Bandini, Laur. a Paladino. Fields generated by torsion poi nts of ellipti c curves 2016 Journal of Number Theory 169: pp. $103-$ 133.

[24] Daniele di Tullio, Manoj Gy awali. Elliptic curves of nearly prime order. pp. 1-19. [Source: iacr.org], access https://eprint.iacr.org/2020/001.pdf

[25] Craig Costello. Com puting Supersingular Isogenies on Kummer Surfaces. Springer. Advances in Cryptology 24 International conference in Theory Cryptography, Part 3.ASIACRYPT 2018. pp. 428-440. 